\title{
Influence of Myofunctional Therapy on Upper Intercanine Distance
}

\author{
Christoph E. Moschik ${ }^{1 *}$, Margit Pichelmayer ${ }^{1}$, Sandra Coulson ${ }^{2}$ and Brigitte Wendl ${ }^{1}$ \\ ${ }^{1}$ Orthodontic Department, School of Dentistry, Medical University Graz, Auenbruggerplatz 12, \\ A-8036 Graz, AT, Austria \\ ${ }^{2}$ Coulson Institute of Orofacial Myology, 2121 South Oneida Street, Suite 625, CO 80224, USA
}

Received: December 15, 2014; Accepted: February 25, 2015; Published: 10 March, 2015

*Corresponding author: Christoph E. Moschik, Orthodontic Department, School of Dentistry, Medical University Graz, Auenbruggerplatz 12 , A-8036 Graz, Austria, Tel: 004-331-638-512-424; Fax: 004-331-638-514-064; E-mail: christoph.moschik@medunigraz.at

\begin{abstract}
Objectives: The study was designed to investigate the correlation between myofunctional exercises, including repositioning of the tongue onto the palate and changes in intercanine distance of the upper jaw in non-growing patients.

Methods: In this retrospective study a total of 141 women and men above the age of 18, treated by the Coulson Institute of Orofacial Myology, with no simultaneous orthodontic therapy, were included. All subjects have participated in a myofunctional therapy program for at least 8 months. The training consisted of various muscle exercises for the lips, cheeks, tongue and body posture and breathing. Additionally, the patients put a small dissolvable gelatine pad onto the palate three times a day to assist the swallowing function. Throughout therapy the length of the philtrum, intercanine distance and overjet were measured.

Results: After 8 sessions the average intercanine distance increased by $3.2 \mathrm{~mm}$, the philtrum elongated by $5.4 \mathrm{~mm}$ and the overjet decreased by $1.2 \mathrm{~mm}$.

Conclusion: The results indicate that tooth position can be changed by muscle therapy, even in non-growing subjects. This implies that muscle training can be a highly supportive therapy for orthodontic treatment.
\end{abstract}

Keywords: Myofunctional; Intercanine distance; Tongue position; Orofacial myology; Arch development

\section{Introduction}

Orofacial myology therapy is a growing field in orthodontic therapy in the last decades around the world. This therapy principle includes the treatment of facial muscle imbalances, training of tongue posture and establishing equilibrium between the tongue, lip and the cheek muscles. Muscle function has become an important concern in orthodontic treatment as the awareness about long-term stability has risen. A balanced facial muscle system is considered as important factor contributing to a stable orthodontic treatment outcome. The intercanine distance is a transverse measurement of the dentition at its most important position for occlusal function, namely the canines. The skeletal, alveolar and dental width of the jaw is determined by two important factors, which are genetics and function.

These two factors correlate with the overall body growth factors and are responsible for morphologic development. While the genetic component currently cannot be influenced or controlled through therapy, it is possible to influence the function. As function is the second most important factor relative to form in the sense of biological and medical terms, it is a logical consequence to design and apply therapy methods indirectly to exert influence on the morphology.

The noun "function" itself is described as "a thing dependent on another factor or factors" by the Oxford dictionary. The function of the stomatognathic system involves the interaction of all participating tissues such as teeth, jaws, temporomandibular joint, muscles and the oral cavity. This concludes that the function of the stomatognathic system is composed of masticatory, phonetic and physiognomic tasks, working together forming a "morphological and functional unit".

When morphology and functions are in balance normal development of the jaws and teeth result, which is called eugnathia. In the case of aberrance from the norm, "dysgnathia", "dysalveolarism" or "malocclusion" and dysfunction occur, it is of high importance to understand, how each of these factors influences the other. Orofacial dysfunction can increase the severity of malocclusion, and vice versa [1,2].

At the beginning of the 20th century, Dr. Edward Angle stated that the field of orthodontics was only at the beginning of understanding what influences the cheeks, tongue and lips have on malocclusion and the difficulties to alter them. Furthermore, he stated that there was no chance to have long-term success in therapy without correcting the malfunction of this system. It is utterly important to recognize and diagnose a certain problem before being able to correct it [3].

Additionally Moyers claimed that therapy was needed to address the root of the problem directly as well as to treat the causes of malocclusion, instead of just straightening teeth [4]. 
Nowadays, the importance of interdisciplinary cooperation has become more vital and orthodontists, myofunctional therapists and logopaedics, specialized physical therapists, earnose-throat specialists, and paediatricians are concerned about the topic of orofacial function.

Each one of these disciplines ought to influence the facial morphology of the patient by changing the function toward a eugnathic norm. This interdisciplinary therapy can be classified as follows:

Orthotropic treatment targets the growth of the face and jaws as well as trying to give the body the chance for self-correction to self-correct. Diagnosis consists of checking the patient for abnormal function, like thumb sucking or mouth breathing. Therapy aims to enable normal swallowing, proper breathing through the nose with a closed mouth and to stop any habits, like thumb sucking or lip chewing. To maximize the effect of this therapy, it is best applied in young children. The earlier therapy is applied and a habit is broken, the more chances there are for the body to return to normal development.

Orthopaedic therapy is applied if jaw discrepancies are already apparent, either due to excessive or restrictive bone growth. It consists of intraoral or extra-oral appliances that apply a high force on the maxillary or mandibular bone and thus attempt to either encourage or decrease growth.

Orthodontic treatment is described as the adjustment of the occlusion by moving and aligning the teeth. In this stage the jaws should be already eugnathic. Orofacial Myofunctional techniques are often only applied after Orthotropics and orthopaedics are finished.

Summarizing these techniques, it can be said that orthotropic therapy should be applied somewhere between ages 0 and 8, at the beginning of the change to the second dentition. This is followed by orthopaedic therapy, which should be executed during the early phase of the second dentition and correlates with the age of 9 until all permanent teeth are exfoliated. By the time the dentition is fully developed, orthodontic therapy is indicated.

As age progresses and growth slowly ceases, the possibility to influence jaw growth decreases. While the impact on jaw growth subsides, tooth movement is still possible throughout the life this is the standard opinion of worldwide orthodontists during the last five decades.

Orofacial myofunctional dysfunction is not solely limited to children, but can also occur in adults with permanent dentition; hence, treatment should be applied whenever improper function occurs. The therapy is then based on the assumption that these changes appear due to improper usage of the oral-musculature system [5] and if an adequate function is restored, the body will adapt and the facial growth will change according to it. This theory goes along with the Functional Matrix Theory [6] which claims that the whole stomatognathic system develops, aside from genetics, along with the demanding functions and the tasks that are given to it.

For example, studies have reported a correlation between the tongue resting position and the form of the palate. If the tongue does not rest on the palate a high vault develops, occasionally narrowing the base of the nose and constricting nasal airflow [7].

Furthermore a correlation between the masseter muscle thickness and the maxillary arch width exists [8]. In general it can be assumed that craniofacial width is linked with the crosssectional areas or thickness of the masticatory muscles [9-11].

By defining the maxillary transverse bone dimension, masticatory muscles can also influence the upper intercanine width, which again can give information about the arch length and inter-molar width [12].

\section{Material and Methods}

The patient population of 141 was fully treated by Sandra Coulson between 1998 and 2012. Requirements of age 18 at treatment start and a minimum of 4 and a maximum of 10 sessions without accompanied orthodontic therapy had to be met. Further it was mandatory, that each patient who was chosen for the study had signed a waiver to acquiesce in a research-study. Therapy itself solely consisted of the facial muscle exercises and putting a gelatine 'spot' about $5 \mathrm{~mm}$ behind the upper incisors onto the palate three times a day.

Patient history files were manually searched at Sandra Coulson's office where a pool of thousands of patient files was available. The search was done by chronically going backwards and any patient who fulfilled the criteria mentioned above was chosen. The selection process stopped with files from 1998, as the recordings were not as meticulous in the older ones. The files were then manually scanned and randomized numbers to ensure anonymity substituted the names. Valuable data was typed into an excel sheet with columns according to the sessions and subdivisions respective to the measured values.

The intercanine distance was measured intraorally by Sandra Coulson herself with a standard calliper in between the tips of the upper canines at each visit of the patient, which is as reliable as measurements on study casts [13]. This parameter was chosen, because the canine-distance is more convenient to measure during the daily clinic routine of an orofacial myologist rather than the intermolar distance. Yet it gives significant information about the dental arch development $[12,14]$.

Upper lip length was measured from the upper to the lower end of the philtrum, where the lip-red begins.

The lower lip length was measured from the outer border of the red-lip line of the lower lip to the beginning of the oral mucosa of the lower lip.

To inquire the influence of time and sex a general linear model with repeated measurements was performed. The calculation was done with the SPSS V.20 software $\left(\right.$ IBM $\left.^{\odot}\right)$ and Microsoft ${ }^{\circledR}$ Excel 2010.

\section{Results}

\section{Patients}

This retrospective study consists of 141 patients, which fulfil 
the former mentioned criteria. 110 of the patients were female, 31 were male and all patients reached at least the age of 18 . Average age was 43.4 years, with a minimum of 18 years and a maximum of 78 years.

\section{Sessions}

Each patient got in average 7.4 sessions in an interval of one month. The amount of participants dropped after the $8^{\text {th }}$ session rapidly and therefore session 9 and 10 were excluded (Table 1).

\section{High vault}

In $20.6 \%$ of the patient cases, there were no data available about the form of the palatal vault. Still $75.9 \%$ patients were classified as high-vault and only $3.5 \%$ patients were classified either as normal or broad vault (Figure 1).

\section{Intercanine width}

After all, the change of intercanine width was highly significant during treatment $(p<0.001)$. There is no significant difference between female and male $(p=0.981)$.Within the first 5 sessions of therapy a rapid increase of inter-canine width was achieved. This was on average $2.7 \mathrm{~mm}$, or $84.4 \%$ of the overall widening. After 8 therapy sessions or the approximate time of 8 months the difference of $3.2 \mathrm{~mm}$ was reached (Figure 1 and Table 2). As there is no significant difference between sexes, in Figure 1 it can be seen that the increase of intercanine width progresses nearly the same until session 3. During the further sessions, there is more fluctuation of the measurements in the male than in the female sample.

\section{Upper lip length}

The upper lip change was highly significant $(p<0.001)$. It increased from $17.0 \mathrm{~mm}$ to $22.4 \mathrm{~mm}$ on average. This is a total of $5.4 \mathrm{~mm}$ within 8 sessions. A steep increase can be seen in Figure 2 after the first session and within approximately one month, the lip length increased $2.9 \mathrm{~mm}$. At about the third session, sometimes a relapse of $1 \mathrm{~mm}$ took place. After that the upper lip length steadily increased at a slower rate than at the beginning. From the seventh session on, the curve flattens and the values remain approximately the same.

Table 1: Number of patients at each session; session 9 and 10 were excluded due to insignificancy.

\begin{tabular}{|l|c|c|}
\hline & Participants & Percentage \\
\hline Session 1 & 141 & $100.00 \%$ \\
\hline Session 2 & 141 & $100.00 \%$ \\
\hline Session 3 & 141 & $100.00 \%$ \\
\hline Session 4 & 141 & $100.00 \%$ \\
\hline Session 5 & 137 & $97.16 \%$ \\
\hline Session 6 & 131 & $92.91 \%$ \\
\hline Session 7 & 116 & $82.27 \%$ \\
\hline Session 8 & 98 & $69.50 \%$ \\
\hline Session 9 & 85 & $60.28 \%$ \\
\hline Session 10 & 73 & $51.77 \%$ \\
\hline
\end{tabular}

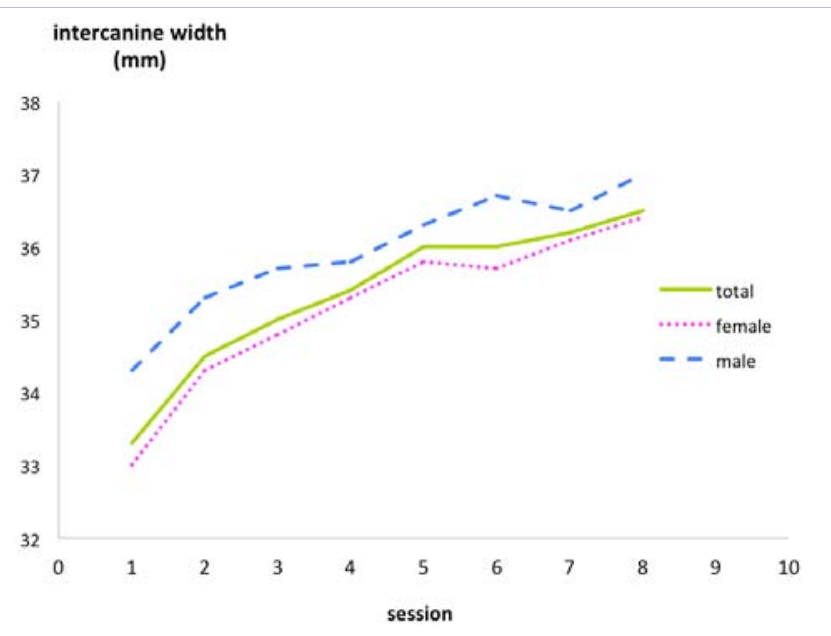

Figure 1: Relation of increase of intercanine width per session in comparison of sex and collective.

There were no significant differences in sexes $(p=0.454)$.

\section{Overjet}

The overjet change was highly significant $(p<0.001)$. It decreased from $2.7 \mathrm{~mm}$ to $1.5 \mathrm{~mm}$ on average throughout 8 sessions. This is a total amount of $1.2 \mathrm{~mm}$. A steep decline of $0.6 \mathrm{~mm}$ can be seen between the first and second sessions and a flattened one in the following six sessions. Also there was a significant difference between female and male measurements $(p<0.001)$. Women's overjet decreased faster from a higher average value of $2.9 \mathrm{~mm}$ and stayed higher throughout the entire treatment time $(p=0.003)$. Only at the end of the therapy the overjet showed the same average value of $1.5 \mathrm{~mm}$ (Figure 3 ).

\section{Discussion}

It has to be mentioned that many of the patients are referred by the general dentist and therefore are preselected. Most of them have already some problems within the stomatognathic system. According to the data, practicing muscle exercises induced changes of the soft tissue through subsequent adaption processes. Muscle tissue has a great capability to adjust its anatomical, histo-chemical, physiological and biochemical properties to meet altered functional requirements [15]. Adaption processes on the anatomical level take place by muscular changes such as in size, cross-sectional diameter or fibre properties [16]. Typically, resistance training of a skeletal muscle, by means of repeated isometric contraction and relaxation, causes an increase in the thickness of the muscle and enhances muscular strength [17]. Notwithstanding that some change might have occurred in the fibres and skin, it can be assumed, that most of the "change" has happened in the facial muscles, especially the orbicularis oris muscle and the tongue. Significant changes in these patterns of the musculature can already be noticed after 3 months of performing exercises. Ingervall [18] reported, that he found significant increase in the bite force and muscle activity after 3 months of chewing exercises.

An increase in volume of the upper lip can be assumed, due 
Table 2: Development of intercanine width according to the sessions.

\begin{tabular}{|c|c|c|c|c|c|c|}
\hline & \multicolumn{2}{|c|}{ Collective } & \multicolumn{2}{|c|}{ female } & \multicolumn{2}{|c|}{ male } \\
\hline & $\begin{array}{c}\text { Arithmetic } \\
\text { average }\end{array}$ & $\begin{array}{l}\text { Standard } \\
\text { deviation }\end{array}$ & $\begin{array}{c}\text { Arithmetic } \\
\text { average }\end{array}$ & $\begin{array}{l}\text { Standard } \\
\text { deviation }\end{array}$ & $\begin{array}{c}\text { Arithmetic } \\
\text { average }\end{array}$ & $\begin{array}{l}\text { Standard } \\
\text { deviation }\end{array}$ \\
\hline Session 2 & 34.5 & 2.2 & 34.3 & 2.1 & 35.3 & 2.1 \\
\hline Session 3 & 35 & 1.8 & 34.8 & 1.6 & 35.7 & 2.2 \\
\hline Session 4 & 35.4 & 1.7 & 35.3 & 1.5 & 35.8 & 2.1 \\
\hline Session 5 & 36 & 1.5 & 35.8 & 1.4 & 36.3 & 1.8 \\
\hline Session 6 & 36 & 1.9 & 35.7 & 2 & 36.7 & 1.4 \\
\hline Session 7 & 36.2 & 1.9 & 36.1 & 1.5 & 36.5 & 2.7 \\
\hline Session 8 & 36.5 & 1.5 & 36.4 & 1.5 & 37 & 1.6 \\
\hline
\end{tabular}

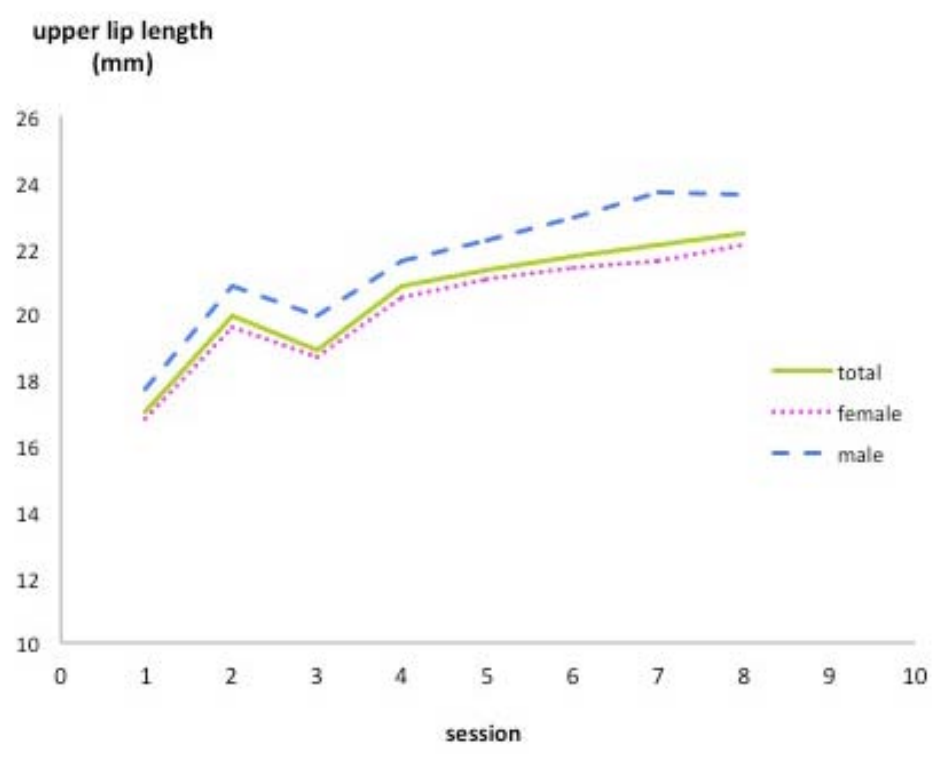

Figure 2: Increase of upper lip length per session in comparison of sex and collective.

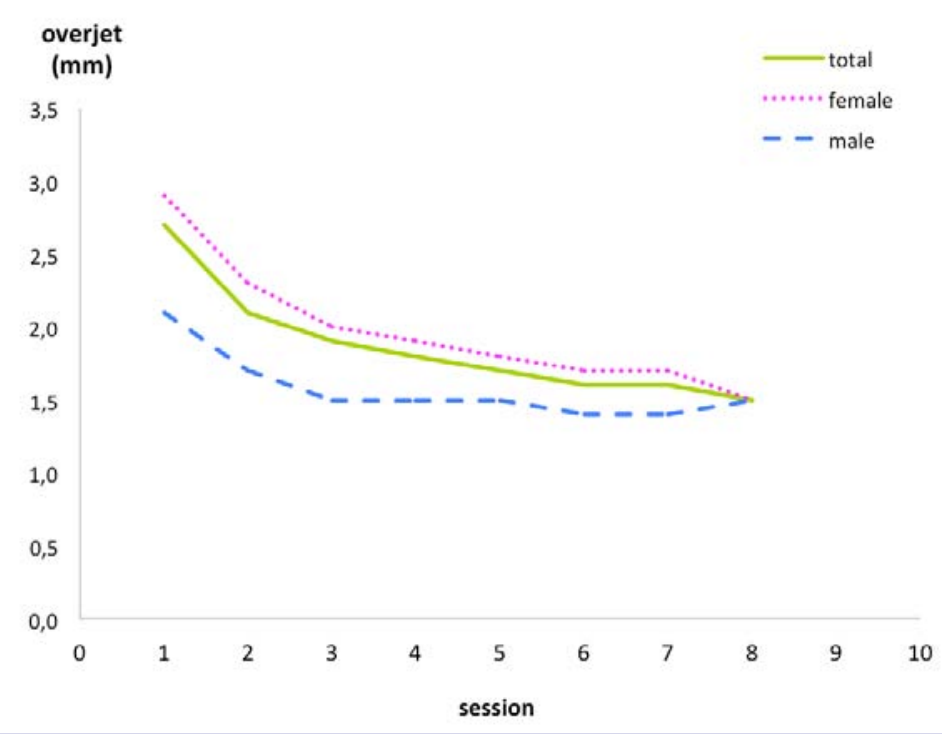

Figure 3: Decrease of overjet per session in comparison of sex and collective. 
to the consistent exercises executed by the patients, which is like "lip jogging". If the volume increases generally, the cross sectional center of the orbicularis oris muscle in the sagittal plane moves slightly away from the incisors.

At the same time the philtrum elongates during therapy, which was indicated by an increase in the upper lip measurement, which was taken in this study. This might have happened due to the mechanical stretching exercises, which increase the collagen synthesis [19]. As a consequence of the elongation of the philtrum, the center of the muscle is lowered in the direction to the occlusal plane. Though the upper lip measurement increased in average about $4 \mathrm{~mm}$, it can be assumed, that the actual lowering of the muscle was less, due to the contour of the lip (Figure 4). If cut in the sagittal plane, the philtrum shows a curve and some of the length contributes to the extent in width and not in length.

Still, these two changes of the resting posture of the upper lip might lead to a slightly increased and more incisally applied force on the incisor crowns, tipping them palatinally. The amount of force does not only depend on the lip posture, but also highly on the inclination and position of the incisors themselves. This was verified in studies, measuring the direct force on the incisors, depending on their inclination and degree of overjet $[20,21]$.

So it is possible, that a decrease of an excessive overjet, as found by measurements in this study, could be a direct result of these changes. As the incisors tip palatinally, they take up more space on the arch in the transverse plane and the position of the canine needs to change as well.

At the same time the tongue is trained to stay on the "spot", slightly behind the palatinal side of the incisors, in the area of the rugae. As the balance between tongue and cheeks defines the tooth position [22], a repositioning of the tongue leads to changes of the force distribution and changes of tooth position should occur. Also, it is reported, that a lowered tongue posture, in patients with myopathies and severe muscle problems, showed a significant increase in the height of the palatal vault and a narrower maxillary arch [23].

In another cross-sectional study of 1065 children between age 5 - 12, Galvez [7] stated that a high palatal vault was present in $85.8 \%$ of children with lip incompetency, in $73.6 \%$ of Class II cases and $65.3 \%$ in Class III cases. As this study was a remittance work, a distinction between the angle classes was not carried out. However, the angle classes apparently had no effect on the treatment results. It is also remarkable that although growth potential is determined by genetics to some degree, function might have some effect on it, even in non-growing patients.

Furthermore, it is shown, that a correlation between a lower rest posture of the tongue, as in Class III patients, and a small upper intercanine width, exists [24]. An inverted rest posture of the tongue, resting on the palate, should therefore, contribute to a wider maxillary intercanine width [25].

In this study a high percentage of patients (75.89\%) showed signs of a high vault. Correlating to the high vault it can be assumed that the maxillary arch was narrow, as presented in previous studies [7]. This leads to the conclusion that in most cases a narrow arch is accompanied by a short intercanine width.

In this study the average intercanine width at the beginning of therapy was $33.3 \mathrm{~mm}$. Similar values are reported for nontreated patients by Paulino [12], Forster [26] and Tibana [27], who also showed that this measurement commonly is stable in adults over a period of 7 years.

Particularly noteworthy are the actual quite tremendous changes of ICW of $3.2 \mathrm{~mm}$ on average after 8 sessions of this muscular therapy. Most of the increase took place in the first 5 sessions, with about $0.5 \mathrm{~mm}$ per session. No study, investigating intercanine width after myofunctional therapy, could be found for comparison.

As x-rays are not available for orofacial myologists, the indicator for incisor movement is solely based on the measurements of overjet. Unfortunately this does not give any information, which teeth, either in the upper arch, lower arch or both have contributed to the measurement changes.

Nevertheless, it is a fact that the upper jaw guides the tooth position of the lower jaw if there is no Crossbite present. Combining this with the increase of the upper intercanine width

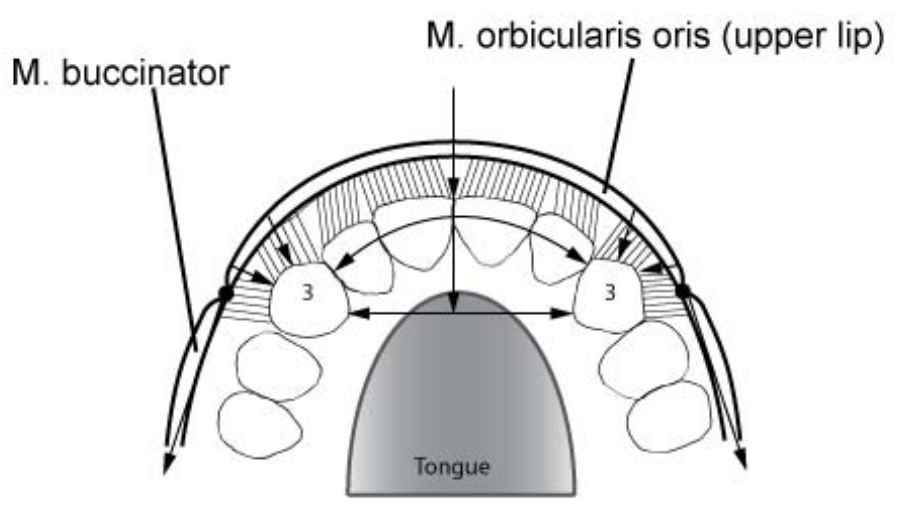

Figure 4: Schematically drawing of the surface relation of the upper incisors and canines exposed to the lip-force after therapy. Contracting forces pushing onto the disto-buccal side of the canine might be relatively small compared to the transmitted, lateral force from the orbicularis oris muscle. 

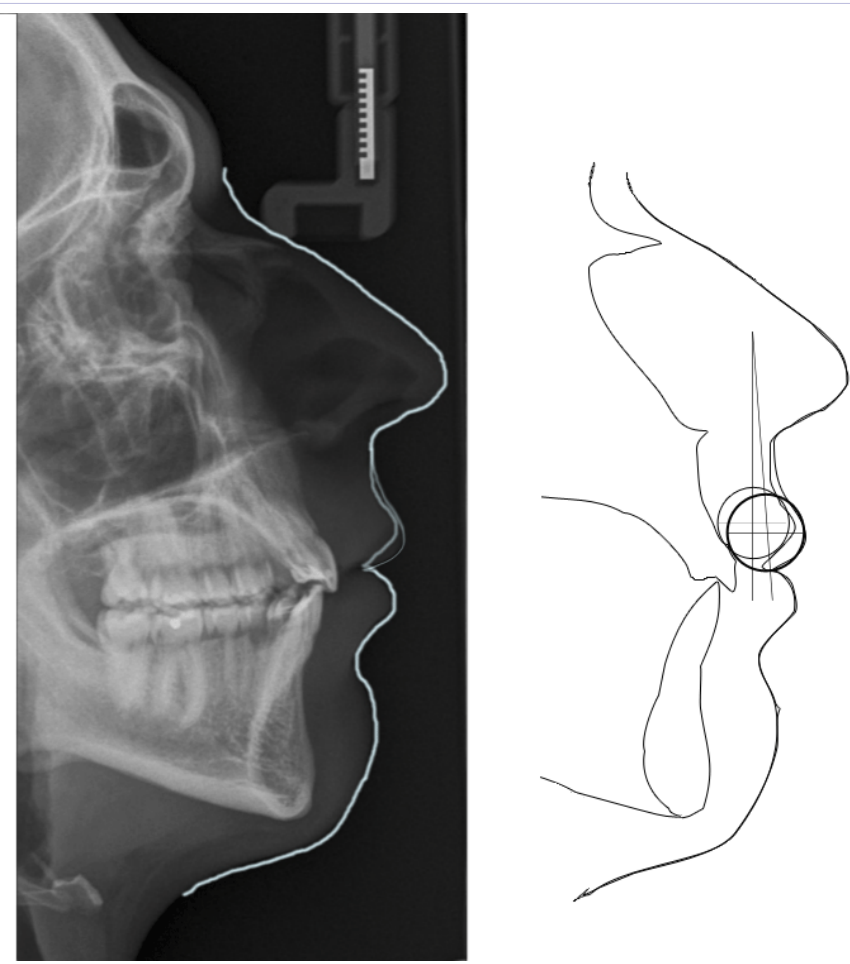

Figure 5: Left: Lateral cephalometric with a photo-shopped superimposition representing the possible change of the lip contour. Right: Schematic drawing of the contours of the cephalometric from the left side and showing the original and moved position of the center of the lip before and after treatment. Upper, thin circle = before therapy. Lower, bold circle = after therapy.

and removing the tongue from a lowered position and stopping a forward pressure of the lower incisors, it can be assumed, that the overjet reduction took place in the upper jaw as well. This could have been achieved by a retro-clination of the upper incisors.

Considering the changes of the upper lip, a possible explanation for these changes might be attained (Figure 5). As the upper lip might have slightly increased the force on the incisors, the position where it is applied changed and, additionally, the counteracting force on the palatinal side of the incisors is reduced at the same time. By repositioning the rest posture of the tongue the incisors might aim for a new equilibrium [21]. This state could be reached with a slight palatinal inclination. Whenever the incisors are retro-clined palatinally, they need more space [28]. If there are no spaces between the front teeth the movement is accompanied by distributing the force from the lips laterally toward the canines.

At the same time, due to the smaller surface and the more distal position, the canines might not be exposed to the pressure of the lip as much as the incisors. As the pressure from the front teeth adds up, it might be enough to move the canines laterally.

Additionally it can be assumed, that the lowest resistance for the canine is on the disto-buccal side and it therefore starts to move to the lateral side. Whether this movement was tipping or bone movement, it cannot be determined by the data available in this study. At the point when the forces of the tongue, lips and cheeks did establish a new equilibrium, e.g. after 5 sessions, tooth movement slowed down, and finally might have ceased.

\section{Conclusion}

A high correlation coefficient between myofunctional therapy in adult patients and intercanine width was found for the upper arch. Repositioning the tongue and practicing muscular exercises for the lips, tongue, face and posture lead to an increase of maxillary intercanine width of $3.2 \mathrm{~mm}$ in average and changes of the upper lip length of $5.4 \mathrm{~mm}$ within roughly eight months.

Changes in the dental formation can therefore be influenced by muscle therapy in non-growing patients.

\section{References}

1. Kittel AM. Myofunktionelle Therapie. Schulz-Kirchner Verlag GmbH. 1997; 1.

2. Melsen B, Attina L, Santuari M, Attina A. Relationships between swallowing pattern, mode of respiration, and development of malocclusion. Angle Orthod. 1987; 57(2): 113-20.

3. Garliner D. Myofunctional Therapy. Saunders Limited. 1976; 1

4. Moyers RE. Handbook of orthodontics. Year Book Medical Pub. 1988; 1.

5. Nakamura A, Zeredo JL, Utsumi D, Fujishita A, Koga Y, Yoshida N. Influence of malocclusion on the development of masticatory function and mandibular growth. Angle Orthod. 2013; 83(5): 749-57.

6. Moss ML. The functional matrix hypothesis revisited. 1. The role of mechanotransduction. Am J Orthod Dentofacial Orthop. 1997; 112(1): $8-11$.

7. Galvez J, Methenitou S. Airway obstruction, palatal vault formation and 
malocclusion: a cross-sectional study. J Pedod. 1989; 13(2): 133-40.

8. Kiliaridis S, Georgiakaki I, Katsaros C. Masseter muscle thickness and maxillary dental arch width. Eur J Orthod. 2003; 25(3): 259-63.

9. Weijs WA, Hillen B. Correlations between the cross-sectional area of the jaw muscles and craniofacial size and shape. Am J Phys Anthropol. 1986; 70(4): 423-31.

10.van Spronsen PH, Weijs WA, Valk J, Prahl-Andersen B, van Ginke FC. Relationships between jaw muscle cross-sections and craniofacial morphology in normal adults, studied with magnetic resonance imaging. Eur J Orthod. 1991; 13(5): 351-61.

11. Hannam AG, Wood WW. Relationships between the size and spatial morphology of human masseter and medial pterygoid muscles, the craniofacial skeleton, and jaw biomechanics. Am J Phys Anthropol. 1989; 80(4): 429-45.

12. Paulino V, Paredes V, Gandia JL, Cibrian R. Prediction of arch length based on intercanine width. Eur J Orthod. 2008; 30(3): 295-8.

13. Ovsenik M. Assessment of malocclusion in the permanent dentition: reliability of intraoral measurements. Eur J Orthod. 2007; 29(6): 654-9.

14. Ricketts RM. Systems RMD. Orthodontic diagnosis and planning 1982; 1

15. Adams GR, Hather BM, Baldwin KM, Dudley GA. Skeletal muscle myosin heavy chain composition and resistance training. J Appl Physiol. 1993; 74(2): 911-5

16. Grünheid T1, Langenbach GE, Korfage JA, Zentner A, van Eijden TM. The adaptive response of jaw muscles to varying functional demands. Eur J Orthod. 2009; 31(6): 596-612.

17. Hather BM, Tesch PA, Buchanan P, Dudley GA. Influence of eccentric actions on skeletal muscle adaptations to resistance training. Acta Physiol Scand. 1991; 143(2): 177-85.

18. Ingervall B, Bitsanis E. A pilot study of the effect of masticatory muscle training on facial growth in long-face children. Eur J Orthod. 1987; 9(1): $15-23$.
19. Jiang C, Shao L, Wang Q, Dong Y. Repetitive mechanical stretching modulates transforming growth factor- $\beta$ induced collagen synthesis and apoptosis in human patellar tendon fibroblasts. Biochem Cell Biol. 2012; 90(5): 667-74

20. Thüer U, Ingervall B. Pressure from the lips on the teeth and malocclusion. Am J Orthod Dentofacial Orthop. 1986; 90(3): 234-42.

21. Proffit WR. Equilibrium theory revisited: factors influencing position of the teeth. Angle Orthod. 1978; 48(3): 175-86.

22. Thüer U, Sieber R, Ingervall B. Cheek and tongue pressures in the molar areas and the atmospheric pressure in the palatal vault in young adults. Eur J Orthod. 1999; 21(3): 29-309.

23. Kiliaridis S, Katsaros C. The effects of myotonic dystrophy and Duchenne muscular dystrophy on the orofacial muscles and dentofacial morphology. Acta Odontol Scand. 1998; 56(6): 369-74.

24. Primozic J, Farcnik F, Perinetti G, Richmond S, Ovsenik M. The association of tongue posture with the dentoalveolar maxillary and mandibular morphology in Class III malocclusion: a controlled study. Eur J Orthod. 2013; 35(3): 388-93.

25. Valdés C, Gutiérrez M, Falace D, Astaburuaga F, Manns A. The effect of tongue position and resulting vertical dimension on masticatory muscle activity. A cross-sectional study. J Oral Rehabil. 2013; 40(9): 650-6.

26. Forster CM, Sunga E, Chung CH. Relationship between dental arch width and vertical facial morphology in untreated adults. Eur J Orthod. 2008; 30(3): 288-94.

27. Tibana RH, Palagi LM, Miguel JA. Changes in dental arch measurements of young adults with normal occlusion--a longitudinal study. Angle Orthod. 2004; 74(5): 618-23.

28. Mah M, Chuan Tan W, Heng Ong S, Huak Chan Y, Foong K. Threedimensional analysis of the change in the curvature of the smiling line following orthodontic treatment in incisor class II division 1 malocclusion Eur J Orthod. 2014; 36(6): 657-64. 